\title{
Recurrent STEMI Precipitated by Marijuana Use: Case Report and Literature Review
}

\author{
Perry Wengrofsky ${ }^{1}$, Ghassan Mubarak ${ }^{1}$, Ahmed Shim ${ }^{2}$, Pramod Theetha Kariyanna ${ }^{2}$, \\ Adam Buzidkowski², Jacob Schwartz ${ }^{1}$, Samy I. McFarlane ${ }^{1, *}$ \\ ${ }^{1}$ Department of Internal Medicine, State University of New York, Downstate Medical Center, Brooklyn, N.Y, U.S.A-11203 \\ ${ }^{2}$ Division of Cardiovascular Disease, Department of Internal Medicine, State University of New York, \\ Downstate Medical Center, Brooklyn, N.Y, U.S.A-11203 \\ *Corresponding author: Samy.mcfarlane@ downstate.edu
}

Received July 08, 2018; Revised August 19, 2018; Accepted September 18, 2018

\begin{abstract}
Marijuana abuse is rapidly growing and currently it is the most common drug of abuse in the United States due to increased legalization for recreational and medicinal use. Delta 9-tetrahydrocannibol, the main psychoactive compound in marijuana, acts via the endocannabinoid system to elicit various cardiovascular physiological effects, and has been associated with many adverse cardiovascular effects such as acute coronary syndrome, arrhythmias, and sudden cardiac death that have previously been reported by our group and others. We present a case of a 30-year-old African-American male with no cardiovascular disease (CVD) risk factors with recurrent ST-segment elevation myocardial infarctions (STEMI) whose coronary angiography revealed recurrent $100 \%$ occlusion of the left anterior descending artery (LAD) in the setting of marijuana smoking. It was the patient's third STEMI with 100\% occlusion of the LAD with each STEMI secondary to thrombosis of a different region of the LAD. Marijuana use was confirmed by urine toxicology screening at each STEMI presentation. Coronary angiography on multiple occasions was negative for stenosis of other epicardial coronary arteries, and coronary calcium scoring was zero. Evaluation for other cardiovascular risk factors including family history of premature coronary artery disease, dyslipidemia, diabetes, and hypercoagulable disorders was negative. Further studies are required to elucidate the mechanisms of marijuana-associated coronary thrombosis and myocardial infarction.
\end{abstract}

Keywords: Marijuana, cannabis, ST-segment myocardial infarction, STEMI

Cite This Article: Perry Wengrofsky, Ghassan Mubarak, Ahmed Shim, Pramod Theetha Kariyanna, Adam Buzidkowsk, Jacob Schwartz, and Samy I. McFarlane, "Recurrent STEMI Precipitated by Marijuana Use: Case Report and Literature Review." American Journal of Medical Case Reports, vol. 6, no. 8 (2018): 163-168. doi: 10.12691/ajmcr-6-8-5.

\section{Introduction}

Marijuana is the most commonly abused illicit drug in the United States, with rates of use expected to rise given increasing legalization for recreational and medical use [1]. Delta 9-tetrahydrocannabinol (THC), the primary psychoactive compound in marijuana, and related cannabinoid agonists interact with the endocannabinoid cannabinoid system through two $\mathrm{G}$ protein-coupled membrane receptors, CBR1 and CBR2, which are diffusely expressed throughout various tissues and organ systems [2]. CBR1 is heavily distributed within the central, peripheral sensory and autonomic nervous systems and is the primary target of THC, mediating the majority of cardiovascular effects via activation of the sympathetic nervous system, but CBR1 receptors are also located in the myocardium and vascular endothelium $[3,4,5]$.

The most common cardiac manifestation of marijuana use in humans is tachycardia, which is attributed to cannabis-mediated activation of sympathetic nervous system activity and inhibition of parasympathetic nervous system activity [6,7]. Additional cardiovascular manifestations of marijuana use include elevation of systolic and diastolic pressures, and increased myocardial oxygen demand and decreased oxygen supply due to elevation of carboxyhemoglobin from inhalation of combustion products $[8,9]$.

Electrophysiologic manifestations, bradyarrhythmia, and tachyarrhythmias have been extensively reported with marijuana use, including atrial fibrillation and flutter $[10,11,12]$, and ventricular tachycardia, fibrillation, and asystole $[13,14,15]$. Marijuana has also been reported to cause acute coronary syndrome, including unstable angina, and acute myocardial infarction, including NSTEMI and STEMI $[16,17,18]$.

We here present a case of recurrent STEMI associated of heavy marijuana smoking with three episodes revealing $100 \%$ occlusion of different segments of the LAD and coronary angiograms showing otherwise patent and nonstenotic arteries. 


\section{Case Presentation}

A 27-year-old African-American male presented with sharp precordial chest pain of six-hour duration with radiations to the left arm. The patient had a smoking history of marijuana where he smoked marijuana at least twice daily since he was a teenager. The patient had a previous history of STEMI at 24 years of age for which he underwent percutaneous coronary intervention (PCI) with balloon angioplasty (Image 1) of the mid-LAD, and was prescribed thereafter but poorly compliant with medication regimen of dual antiplatelet therapy (DAPT) with aspirin and prasugrel, high intensity atorvastatin, metoprolol succinate, and enalapril. Family history was not significant for coronary artery disease or sudden cardiac death.

Upon presentation, patient was hemodynamically stable. 12 lead electrocardiogram (ECG) revealed sinus rhythm at a rate of $72 \mathrm{bpm}$ with ST segment elevation (STE) in limb leads II, III, and aVF and in precordial leads V3-V6 (Image 2). Serial troponin levels were initially negative but uptrended to a peak of 39.46 and subsequently downtrended.
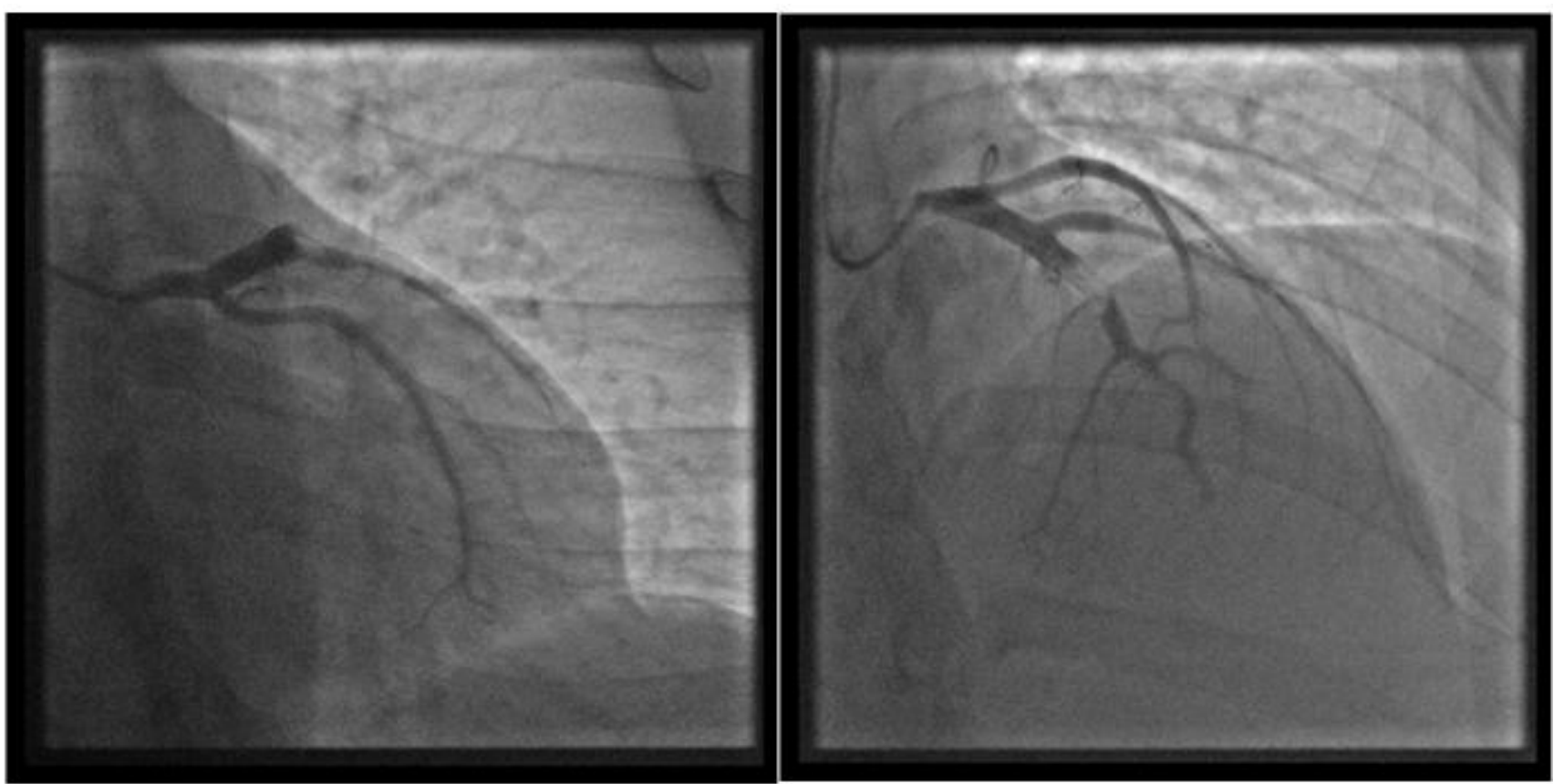

Image 1. Patient's first STEMI at 24 years of age for which he underwent PCI with balloon angioplasty. Angiography showing occlusive filling defect compatible with thrombotic occlusion of the mid LAD pre-angioplasty (left) and post-angioplasty (right)

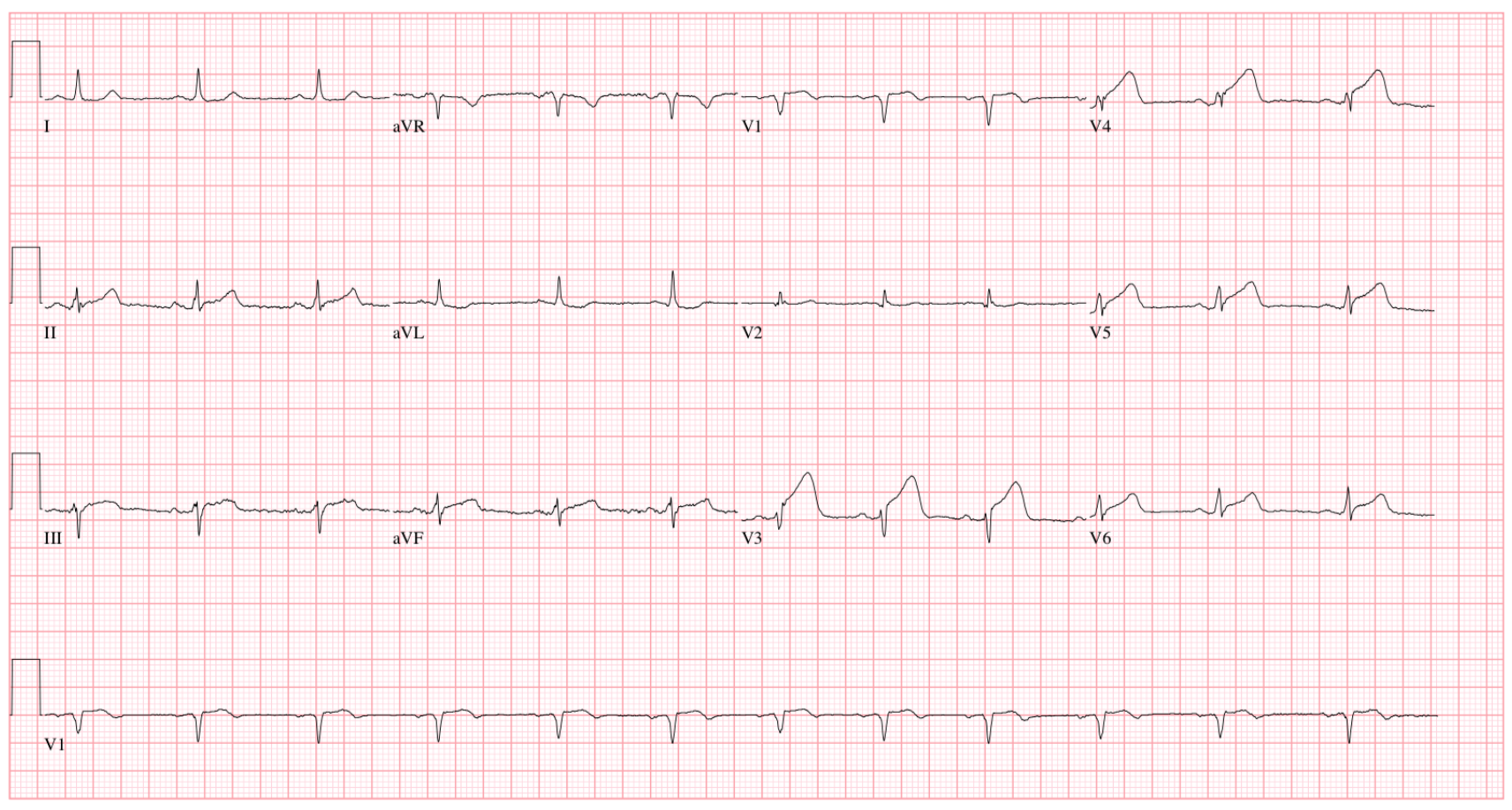

Image 2. ECG on presentation at age 27, significant for STE in II, III, aVF, and V3 -V6 

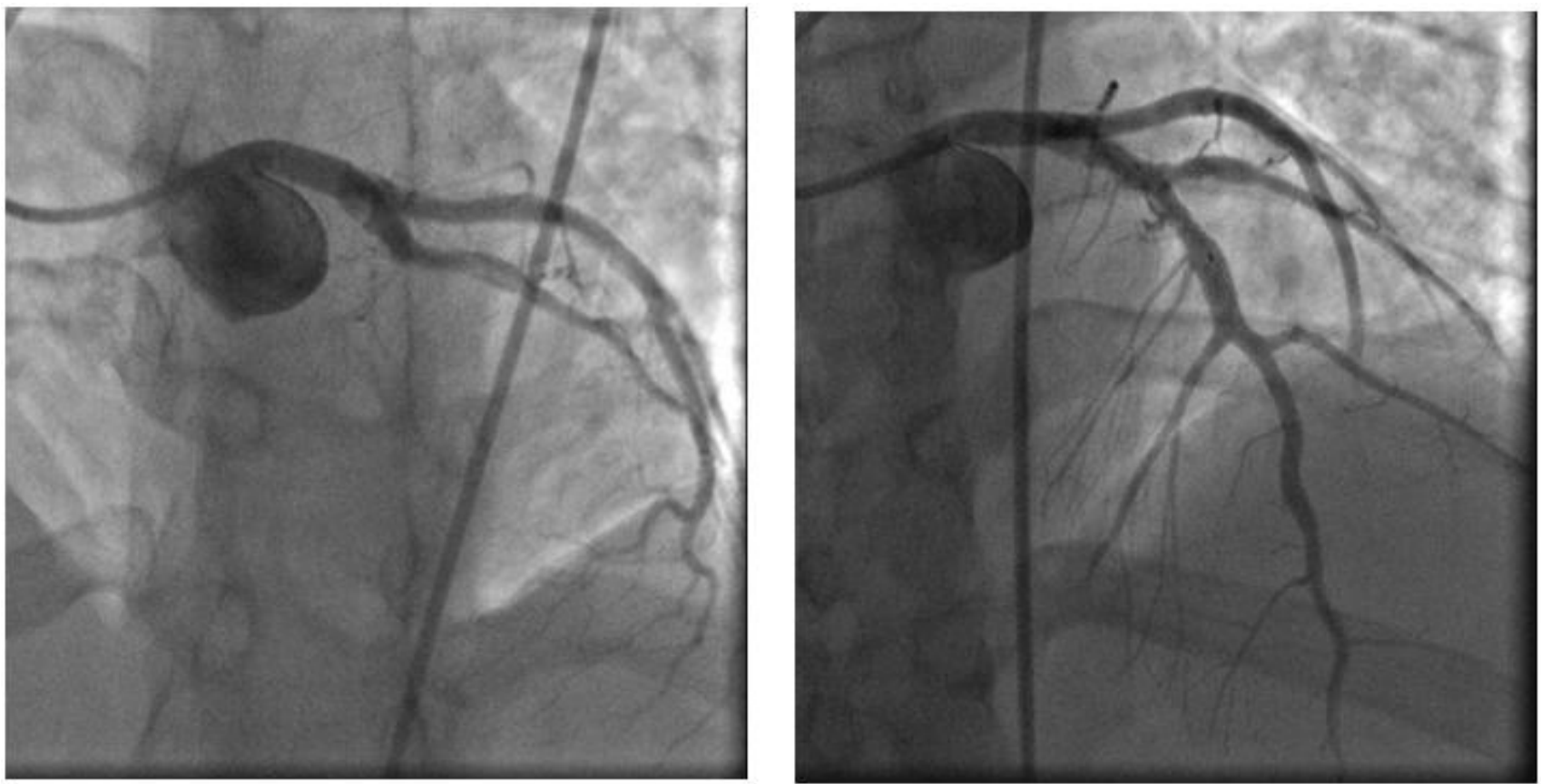

Image 3. $2^{\text {nd }}$ STEMI at 27 years of age for which he underwent PCI with balloon angioplasty of LAD, with angiography showing occlusive filling defect compatible with thrombotic occlusion of the mid LAD pre-balloon angioplasty (left) and post-balloon angioplasty (right)

The patient was loaded with aspirin, clopidogrel, and heparin, and underwent PCI with balloon angioplasty of a $100 \%$ mid-LAD stenosis (image 3). Urine toxicology was positive for THC. 2D Transthoracic echocardiography (TTE) showed distal septal, anterior, and apical hypokinesis with reduced ejection fraction estimated at $40 \%$. Patient was subsequently discharged on aspirin, prasugrel, atorvastatin, metoprolol succinate, and enalapril.

The patient represented at 30 years of age with sudden onset precordial sharp nonradiating chest pain that awoke him from sleep. The pain was associated with intermittent shortness of breath and nausea. The patient endorsed episodes of chest pain with exertion at least once a month prior to his presentation, episodes of chest pain that usually lasted 5 minutes and were relieved with rest. Patient endorsed continuing to smoke marijuana twice a day. Patient had undergone coronary calcium scan a few months prior to presentation with a cardiac calcium score of zero.

Upon presentation, patient was hemodynamically stable. 12 lead ECG revealed sinus rhythm at a rate of $68 \mathrm{bpm}$ with STE in V2-V4 (Image 4). Serial troponins were initially negative but uptrended to a peak of 90.94 prior and subsequently downtrended. Urine toxicology was positive for THC.

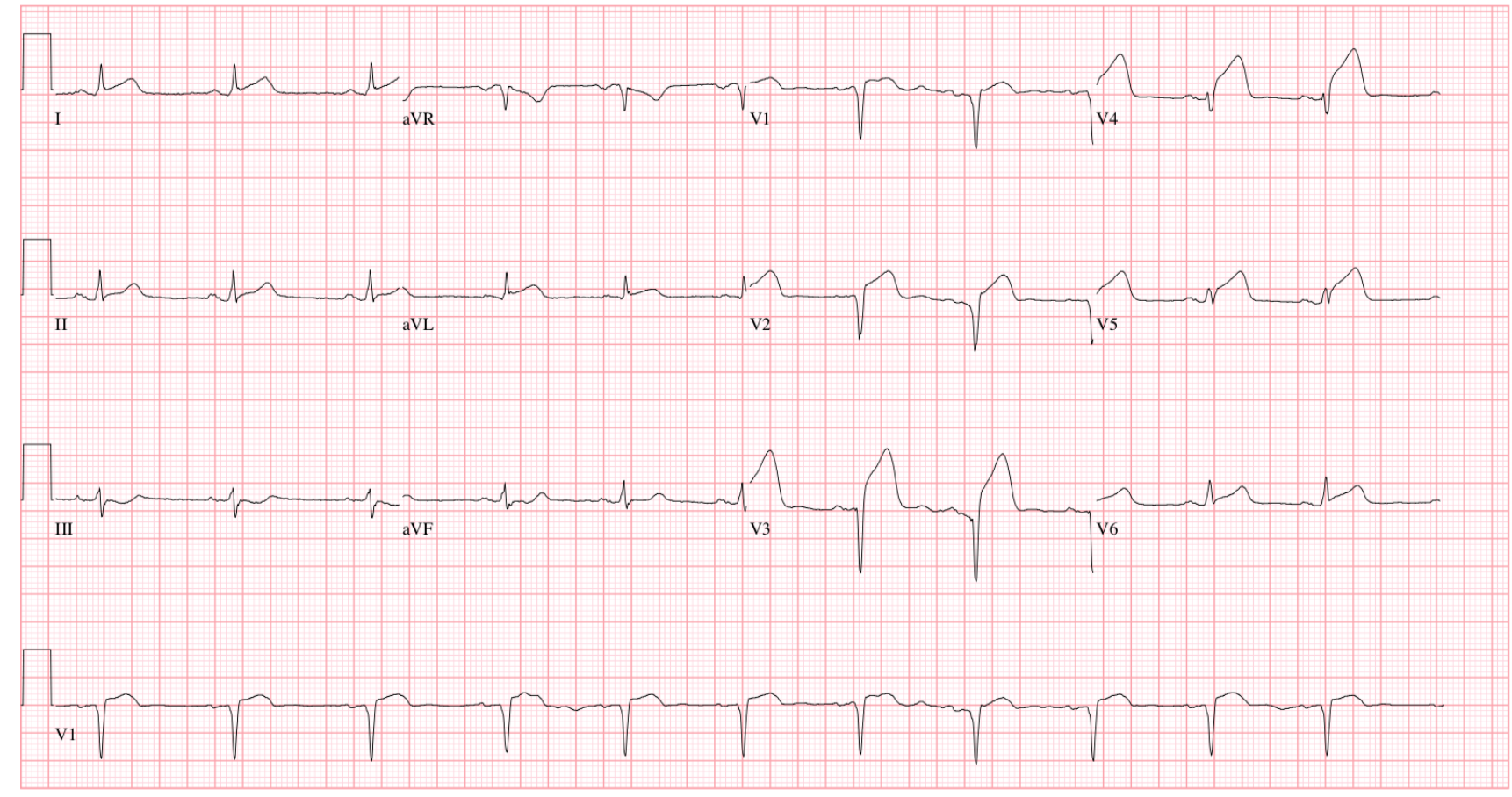

Image 4. ECG on presentation at age 30, significant for STE in V2-V4 

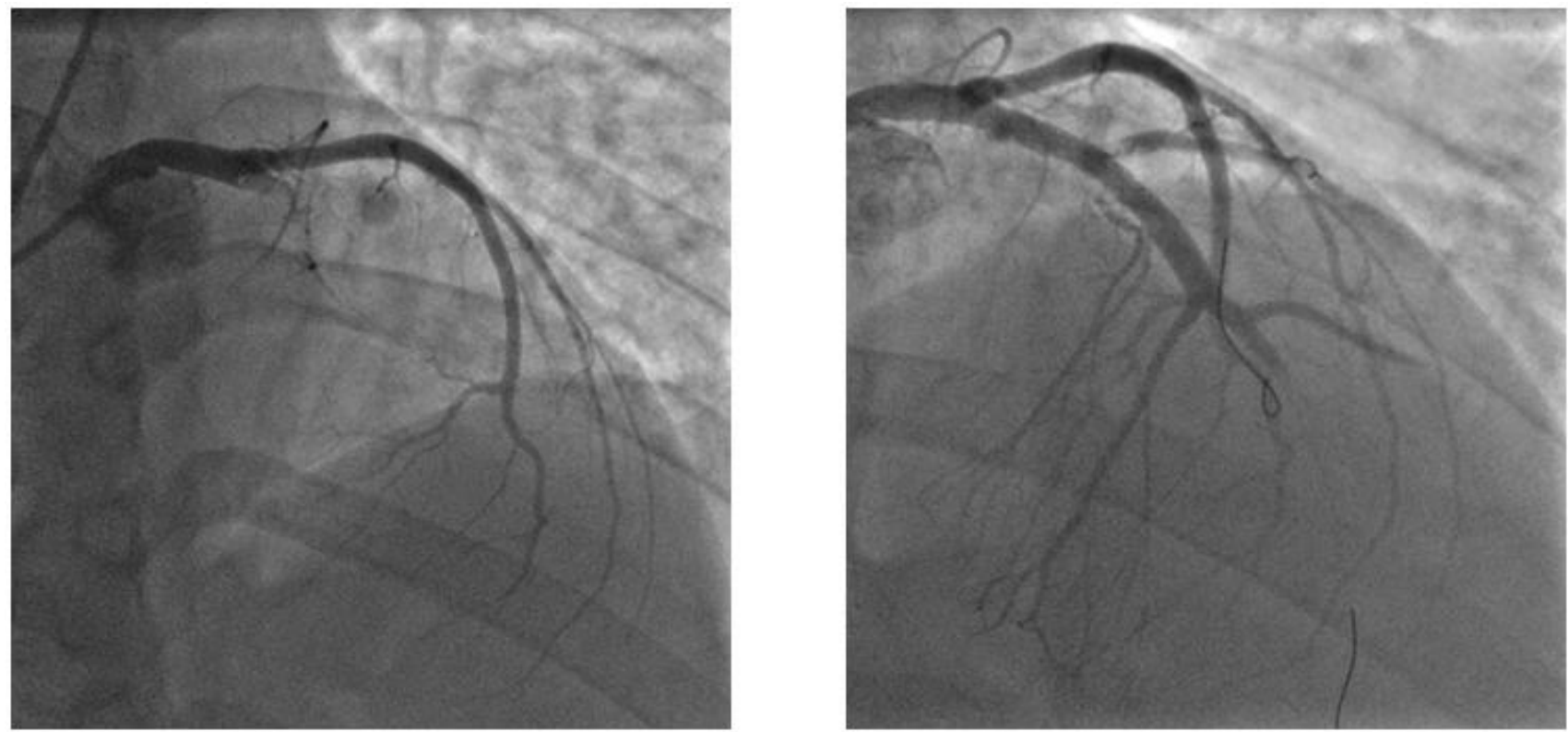

Image 5. $3^{\text {rd }}$ STEMI at age 30 for which he underwent PCI with deployment of DES, with angiography showing occlusive filling defect compatible with thrombotic occlusion of the proximal LAD (left) and restored LAD flow upon deployment of DES (right)

Patient was loaded with aspirin and ticagrelor, and underwent PCI with deployment of a drug eluting stent (DES) in the proximal LAD. PCI was complicated by hypotension and ventricular tachycardia, for which patient was commenced on norepinephrine and amiodarone drips and an intra-aortic balloon pump was placed with concurrent 48-hour heparin infusion.

TTE showed reduced ejection fraction estimated at 20$25 \%$, and severe hypokinesis of large areas of the anterior, anteroseptal, and apical walls. Patient was subsequently discharged on continued DAPT, beta-blocker, high intensity statin, and given his reduced EF in the setting of ischemic cardiomyopathy, transitioned from enalapril to sacubitrilvalsartan.

\section{Discussion}

Marijuana (cannabis) and related synthetic cannabinoid receptor agonist associated cardiovascular events have been extensively reported in the literature. Multiple isolated case reports of marijuana associated acute myocardial infarction, including NSTEMI and STEMI, have been previously documented in the literature $[16,17,18,19,20]$. We present the case of a young male without underlying cardiovascular risk factors with a longstanding heavy marijuana smoking history and recurrent STEMIs with angiographically normal coronary arteries. To our knowledge, this is the first case report of recurrent STEMI associated with marijuana use. Given the increasing prevalence of marijuana use in the US, understanding the adverse effects of marijuana on the cardiovascular system, in particular the risk of acute myocardial infarction, is of great importance.

Marijuana has been shown to increase the risk of myocardial infarction nearly five times within 60 minutes after marijuana use and confers a 1.5-3\% annual risk of an acute cardiovascular event [21]. Cannabis associated myocardial infarction has most commonly been reported in young men without a previous history of coronary artery disease (CAD) and normal coronary angiograms $[22,23,24]$. Analysis of the diverse hemodynamic, physiologic, and hematologic effects stimulated by cannabis and related cannabinoid receptor agonists illuminates the various pathophysiological processes behind cannabis consumption and acute myocardial infarction.

CBR1 activation, as the predominant endocannabinoid receptor within the myocardium, vascular endothelium, and neurons responsible for autonomic regulation of the cardiovascular system, increases cardiac workload and oxygen demand via tachycardia [6,7,25], increased afterload from elevated systolic blood pressure [26]. Oxygen demand-supply mismatch contributing to myocardial ischemia is due to decreased oxygen supply from elevated levels of carboxyhemolgobin, nearly five times higher than seen in tobacco cigarettes, generated from inhalation of combustion products $[8,9,21,27]$. Cannabis derived reactive oxygen species, CBR1 mediated endothelial dysfunction, and regional arterial vasospasm cause ischemia and increase the risk for subtotal to total arterial occlusion and downstream infarction $[28,29]$. The cannabinoid vasoactive effect ranges from vasoconstriction to vasodilatation in different vascular beds, with the coronary arterial bed likewise demonstrating both vasoconstriction and vasodilation [7,30]. The interplay of endothelial dysfunction and discrepant vasoconstriction and vasodilation causes ischemia and infarction with typical coronary angiographic findings of slow coronary flow phenomenon [31,32]. Interestingly, coronary angiography for our patient revealed TIMI grade 0 flow (no flow) on cardiac catheterization during his second and third STEMI, suggestive of filling defects consistent with coronary thrombus and highlighting the crucial role of THC mediated thrombosis.

CBR1 and CBR2 also play an important role in the precipitation of acute myocardial infarction via their distribution on circulating blood cells including monocytes, macrophages, and platelets [33]. THC prolongation lipopolysaccharide-stimulated tissue factor protein expression in activated monocytes generates a THC dose-dependent procoagulant effect $[34,35]$. Platelet exposure to THC has 
been shown to increase platelet membrane glycoprotein IIb-IIIa and P-selectin in a similar dose-dependent manner [36]. Furthermore, cannabinoid metabolites anandamide and 2-arachidonoylglycerol have been demonstrated to activate platelets [37].

In summary, we present a case of marijuana induced recurrent STEMI in acknowledged heavy marijuana smoking with multiple coronary angiograms demonstrating acute single vessel filling defects consistent with thrombus without atherosclerotic narrowing of other coronary arteries. Although long-term cannabis smoking has been shown to not be associated with increased cardiovascular risk, it has been shown to dramatically increase the acute risk of myocardial infarction, as acute as within the first hour after consumption [21,38]. Given the vasoactive, procoagulant, and prothrombotic properties of THC, further studies are needed to understand how these entities and their important roles in the pathophysiology of acute myocardial infarction specifically interact in THC induced myocardial infarction.

\section{Acknowledgements}

This work is supported, in part, by the efforts of Dr. Moro O. Salifu M.D., M.P.H., M.B.A., M.A.C.P., Professor and Chairman of Medicine through NIH Grant number S21MD012474.

\section{References}

[1] Center for Behavioral Health Statistics and Quality (CBHSQ). Behavioral Health Trends in the United States: Results from the 2014 National Survey on Drug Use and Health. Rockville, MD: Substance Abuse and Mental Health Services Administration; 2015. HHS Publication No. SMA 15-4927, NSDUH Series H-50.

[2] Gelfand EV, Cannon CP. Rimonabant: a cannabinoid receptor type 1 blocker for management of multiple cardiometabolic risk factors. Journal of the American College of Cardiology. 2006; 47: 1919-1926.

[3] Benowitz NL, Rosenberg W, Rogers W, et al. Cardiovascular effects of intravenous delta-9-tetrahydrocannabinol: autonomic nervous mechanisms. Clinical Pharmacology \& Therapies. 1979; 25: 440-446.

[4] Bonz A, Laser M, Küllmer S, et al. Cannabinoids acting on CB1 receptors decrease contractile performance in human atrial muscle. Journal of Cardiovascular Pharmacology. 2003; 41: 657-664.

[5] Liu J, Gao B, Mirshahi F, et al. Functional CB1 cannabinoid receptors in human vascular endothelial cells. Biochemical Journal. 2000; 346 Pt 3: 835-840.

[6] Kanakis C Jr, Pouget JM, Rosen KM. The effects of delta-9tetrahydrocannabinol (cannabis) on cardiac performance with and without beta blockade. Circulation. 1976; 53: 703-707.

[7] Beaconsfield P, Ginsburg J, Rainsbury R. Mariuana smoking. Cardiovascular effects in man and possible mechanisms. New England Journal of Medicine. 1972; 287: 209-212.

[8] Aronow WS, Cassidy J. Effect of marihuana and placebomarijuana smoking on angina pectoris. New England Journal of Medicine. 1974; 291: 65-67.

[9] Wu TC, Tashkin DP, Djahed B, et al. Pulmonary hazards of smoking marijuana as compared with tobacco. New England Journal of Medicine. 1988; 318: 347-351.

[10] Korantzopoulos P, Liu T, Papaioannides D, et a. Atrial fibrillation marijuana smoking. International Journal of Clinical Practice. 2008; 62(2): 308-313.

[11] Lehavi A, Shay M, Gilony C, et al. Marijuana smoking and paroxysmal atrial fibrillation. Harefuah. 2005; 144(1): 2-3.

[12] Fisher BA, Ghuran A, Vadamalai V, et al. Cardiovascular complications induced by cannabis smoking: a case report and review of the literature. Emergency Medicine Journal. 2005: 22(9): 679-680.

[13] Baranchuk A, Johri AM, Simpson CS, et al. Ventricular fibrillation triggered by marijuana use in a patient with ischemic cardiomyopathy: a case report. Cases Journal. 2008; 1(1): 373.

[14] Diffley M, Armenian P, Gerona R, et al. Catecholaminergic polymorphic ventricular tachycardia found in an adolescent after a methylenedioxymethampthetamine and marijuana-induced cardiac arrest. Critical Care Medicine. 2012: 40(7): 2223-2226.

[15] Brancheau D, Blanco J, Gholkar, et al. Cannabis induced asystole. Journal of Electrocardiology. 2016; 49(1): 15-17.

[16] Hodcroft CJ, Rossiter MC, Buch AN, et al. Cannabis-associated myocardial infarction in a young man with normal coronary arteries. Journal of Emergency Medicine. 2014; 47(3):277-281.

[17] Mills B, Dishner E, Velasco CE. Acute myocardial infarction triggered by use of synthetic cannabis. Baylor University Medical Center Proceedings. 2018; 31(2):200-20.

[18] Ghannem M, Belhadi I, Zaghdoudi M, et al. [Cannabis and acute coronary syndrome with ST segment elevation]. Annales De Cardiologie Et D'angeiologie [serial online]. 2013; 62(6). 424-428

[19] Velibey Y, Sahin S Tanik O, et al. Acute myocardial infarction due to marijuana smoking in a young man: gyuilty should not be underestimated. American Journal of Emergency Medicine. 2015; 33(8): 1114.e1-e3.

[20] Safaa AM, Markham R, Jayasinghe R. Marijuana-induced recurrent acute coronary syndrome with normal coronary angiograms. Drug and Alcohol Review. 2012;31:91-94

[21] Mittelman MA, Lewis RA, Maclure M, et al. Triggering myocardial infarction by marijuana. Circulation. 2001; 103:28052809.

[22] Caldicott DG, Holmes J, Roberts-Thomson KC, et al. Keep off the grass: marijuana use and acute cardiovascular events. European Journal of Emergency Medicine. 2005; 12: 236-244.

[23] Jouanjus E, Raymond V, Lapeure-Mestre M, et al. What is the current knowledge about the cardiovascular risk for users of cannabis based products? A systematic review. Current Atherosclerosis Reports. 2017; 19:26

[24] Lee J, Sharma N, Saladini Aponte C, et al. Clinical characteristics and angiographic findings of myocardial infarction among marijuana users and non-users. Scifed Journal of Cardiology. 2017 1(2): 1000008.

[25] Sulkowski A, Vachon L, Rich E. Propanolol effects on acute marihuana intoxication in man. Psychopharmacology. 1977; 52: 47-53.

[26] Alshaaraway O, Elbaz HA. Cannabis use and blood pressure levels: United States Health and Nutrition Examination Survey, 2005-2012. Journal of Hypertension. 2016; 34: 1507-1512.

[27] Wu TC, Tashkin DP, Djahed B, et al. Pulmonary hazards of smoking marijuana as compared with tobacco. New England Journal of Medicine. 1988; 318(6): 347-351.

[28] Lipina C, Hundal HS. Modulation of cellular redox homeostasis by the endocannabinoid system. Open Biology. 2016; 6: 150276.

[29] Singh A, Saluja S, Kumas A, et al. Cardiovascular complications of marijuana and related substances: A Review. Cardiology and Therapy. 2018; 7: 45-59.

[30] Quercioli A, Pataky Z, Vincenti G, et al. Elevated endocannabinoid plasma levels are associated with coronary circulatory dysfunction in obesity. European Heart Journal. 2011; 32: 1369-1378.

[31] Khouzam RN, Kabra R, Soufi MK. Marijuana, bigeminal premature ventricular contractions and sluggish coronary flow: are they related? Journal of Cardiology Cases. 2013; 8(4): 121-124.

[32] Rezkalla SH, Sharma P, Kloner RA. Coronary no-flow and ventricular tachycardia associated with habitual marijuana use. Annals of Emergency Medicine. 2003; 42(3): 365-369.

[33] Pacher P, Steffens S, Haskó G, et al. Cardiovascular effects of marijuana and synthetic cannabinoids: the good, the bad, and the ugly. Nature Reviews Cardiology. 2018;15(3):151-166.

[34] Dahdouh Z, Roule V, Lognoné, et al. cannabis and coronary thrombosis: what is the role of platelets? Platelets. 2012; 23: 243-245.

[35] Williams JC, Klein TW, Goldberger BA, et al. $\Delta(9)$ Tetrahydrocannabinol (THC) enhances lipopolysaccharidestimulated tissue factor in human monocytes and monocytederived microvesicles. Journal of Inflammation (London). 2015; 12: 39 . 
[36] Deusch E, Kress HG, Kraft B, et al. The procoagulatory effects of delta-9-tetrahydrocannabinol in human platelets. Anastheia \& Analgesia. 2004; 99: 1127-1130.

[37] Randall MD. Endocannabinoids and the haematological system. British Journal of Pharmacology. 2007; 152: 671-675.
[38] Rodondi N, Pletcher MJ, Liu K, et al. Marijuana use, diet, body mass index, and cardiovascular risk factors (from the CARDIA study). American Journal of Cardiology. 2006; 98: 478-484. 